Original Research Paper

\title{
Convergence of Renormalization Group Transformations of Gibbs Random Field
}

\author{
${ }^{1}$ Farida Kachapova and ${ }^{2}$ Ilias Kachapov \\ ${ }^{1}$ Auckland University of Technology, Auckland, New Zealand \\ ${ }^{2}$ University of Auckland, Auckland, New Zealand
}

\author{
Article history \\ Received: 13-03-2016 \\ Revised: $13-05-2016$ \\ Accepted: 13-05-2016 \\ Corresponding Author: \\ Farida Kachapova \\ Auckland University of \\ Technology, Auckland, New \\ Zealand \\ Email: farida.kachapova@aut.ac.nz
}

\begin{abstract}
Statistical mechanics describes interaction between particles of a physical system. Particle properties of the system can be modelled with a random field on a lattice and studied at different distance scales using renormalization group transformation. Here we consider a thermodynamic limit of a lattice model with weak interaction and we use semi-invariants to prove that random fields transformed by renormalization group converge in distribution to an independent field with Gaussian distribution as the distance scale infinitely increases; it is a generalization of the central limit theorem to weakly dependent fields on a lattice.
\end{abstract}

Keywords: Gibbs Measure, Renormalization Group, Semi-Invariant, Thermodymanic Limit, Weak Dependence

\section{Introduction}

The classical Central Limit Theorem (CLT) considers a sequence of independent random variables and their normalized sums. Here we consider a sequence of weakly dependent random fields on a multi-dimensional integer lattice. We are interested in the limiting distribution of normalized sums of these variables, similar to the sums in the classical CLT. Such problems arise in the research of Renormalization Group (RG) in statistical mechanics.

The concept of $\mathrm{RG}$ as a scale transformation was introduced and studied in works of Kadanoff (Kadanoff, 1966; Kadanoff, 2013), Wilson and Kogut (1974). Originally RG was defined in terms of Hamiltonian (interpreted as the interaction potential). A rigorous formula of the renormalized Hamiltonian was derived by Kashapov (1980). Bertini et al. (1999) and Lorinczi et al. (1998) studied Gibbs property of the renormalized Hamiltonian.

Other research on RG are based on limit theorems of probability theory. Sinai (1976) studied distributions invariant under the RG transformation and showed that Gaussian distribution is one of them. Newman (1980) proved the CLT on an integer lattice under Fortuin-Kasteleyn-Ginibre (FKG) conditions. Bolthausen (1982) proved the CLT on an integer lattice under some strong conditions.

In this paper we study the limiting distribution of Gibbs random field under the RG transformations and we improve our results from (Kachapova and Kachapov, 2015). We show that under the condition $|\lambda|<C$ the limiting distribution in a high-temperature region is an independent Gaussian distribution. The novelty of our result is in finding a broad condition for the interaction parameter $\lambda$, for which the CLT on a lattice holds; this condition is $|\lambda|<C$ for a constant $C$ depending only on the lattice dimension. This is a simple condition and is easy to check and it is stated in a form preferable for physicists, without tedious technical details.

The FKG conditions in the Newman's version of CLT (Newman, 1980) do not always hold; for example, they hold for the ferromagnetic Ising model but not for the anti-ferromagnetic one; while our theorem covers both models and more.

The conditions in the Bolthausen's theorem (1982) involve supremum of probabilities and supremum of covariances, which are difficult to estimate. Also Bolthausen proved his theorem under the assumption of absolute convergence of three series and positivity of a fourth series, while in our paper we prove convergence of all necessary series. In his theorem Bolthausen did not consider RG transformations but only normalized sums of a random variable on finite sets and he proved convergence in distribution of these sums to a single random variable. In our paper we prove convergence in distribution of RG transformations of a random field to another random field.

Mathematicians doing research in statistical mechanics try to create the mathematical structures that make foundation of physical theories. They appreciate rigorous, non-contradictory and transparent theories. 
They also make effort to obtain simplest possible proofs for existing theorems. In our paper we use a new approach in proving the CLT for weakly dependent random fields; this approach is based on estimation of semi-invariants.

We apply the techniques of Malyshev and Minlos (1991; Malyshev, 1980) to estimate semi-invariants of a random field and we use these estimations to prove a generalization of the CLT to weakly dependent random fields on a lattice.

Semi-invariants are synonyms for cumulants and Ursell functions. We give the definition of semiinvariants and briefly describe their properties in section 2. In section 2 we also introduce other necessary concepts from probability theory and statistical mechanics and briefly prove some relevant lemmas.

In section 3 we state the main result of this paper: the central limit theorem for Gibbs random field transformed by $R G$, with a brief discussion of its meaning.

The rest of the paper develops techniques for proving the main theorem. In particular, in section 4 we prove an inequality about the number of links in a set with a symmetric binary relation and apply it to estimate semiinvariants of a random field (Estimation Theorem).

In section 5 we prove the main theorem. In subsections 5.1 and 5.2 we prove a series of lemmas, which lead to the direct proof of the main theorem in subsections 5.3 and 5.4. In particular, we find an expression for the limiting variance and show the equality to 0 of all other limiting semi-invariants of the random field transformed by $\mathrm{RG}$. We complete the proof of the main theorem by applying Carleman theorem to the limiting distribution.

\section{Main Concepts}

\subsection{Semi-Invariants}

Denote $E(X)$ the expectation of a random variable $X$. Semi-invariant is a generalization of the concepts of expectation and covariance. The following is a slight modification of the definition in (Malyshev and Minlos, 1991), pg. 27-33.

\section{Definition 2.1}

Suppose $X_{1}, \ldots, X_{m}$ are random variables on the same probability space and $M=\{1,2, \ldots, m\}$ is the set of their indices. For any $S \subseteq M$, we denote $X_{S}=\prod_{i \in S} X_{i}$. We assume that the expectation of every such product is finite.

A semi-invariant of random variables $X_{1}, \ldots, X_{m}$ is:

$$
\left\langle X_{1}, \ldots, X_{m}\right\rangle=\sum_{\alpha}(-1)^{k-1}(k-1) ! E\left(X_{1}\right) \ldots E\left(X_{m}\right)
$$

where the sum is taken over all partitions $\alpha=\left\{S_{1}, \ldots\right.$, $\left.S_{k}\right\}$ of the set $M$. By a partition we mean a set of disjoint, non-empty subsets of $M$ such that their union equals $M$.

\section{Notation}

If $I=\left(i_{1}, \ldots, i_{m}\right)$ is a sequence or a set of indices, we denote $\left\langle X_{I}^{\prime}\right\rangle=\left\langle X_{i_{1}}, \ldots, X_{i_{m}}\right\rangle$.

Semi-invariants characterize the distribution and dependence of random variables. Other terms for a semiinvariant are cumulant and Ursell function.

\section{Example 2.1}

Suppose $X, X_{1}, X_{2}$ and $X_{3}$ are random variables. Denote $\mu$ the expectation of $X$ and $\sigma$ the standard deviation of $X$. Then the following hold:

1) $\langle X\rangle=\mu$.

2) $\left\langle X_{1}, X_{2}\right\rangle=\left\langle X_{1} X_{2}\right\rangle-\left\langle X_{1}\right\rangle\left\langle X_{2}\right\rangle=\operatorname{cov}\left(X_{1}, X_{2}\right)$, the covariance of $X_{1}$ and $X_{2}$.

3) $\left\langle X_{1}, X_{2}, X_{3}\right\rangle=\left\langle X_{1} X_{2} X_{3}\right\rangle-\left\langle X_{1}\right\rangle\left\langle X_{2} X_{3}\right\rangle-\left\langle X_{2}\right\rangle\left\langle X_{1} X_{3}\right\rangle$ - $\left\langle X_{3}\right\rangle\left\langle X_{1} X_{2}\right\rangle+2\left\langle X_{1}\right\rangle\left\langle X_{2}\right\rangle\left\langle X_{3}\right\rangle$.

4) $\langle X, X\rangle=\sigma^{2}$, the variance of $X$.

5) $\langle X, X, X\rangle / \sigma^{3}$ equals the skewness of $X$.

6) $\langle X, X, X, X\rangle / \sigma^{4}$ equals the kurtosis of $X$.

\section{Lemma 2.1}

1. A semi-invariant is a symmetrical and multi-linear functional on random variables

2. If $0<n<m$ and two random vectors $\left(X_{1}, \ldots, X_{n}\right)$ and $\left(X_{n+1}, \ldots, X_{m}\right)$ are independent of each other, then $\left\langle X_{1}, \ldots, X_{n}, X_{n+1}, \ldots, X_{m}\right\rangle=0$.

3. For set $M=\{1,2, \ldots, m\}$ :

$$
E\left(X_{M}\right)=\left\langle X_{M}\right\rangle=\sum_{\alpha}\left\langle X_{S_{1}}^{\dagger}\right\rangle \ldots\left\langle X_{S_{k}}^{\dagger}\right\rangle,
$$

where the sum is taken over all partitions $\alpha=\left\langle S_{1}, \ldots, S_{k}\right\rangle$ of the set $M$.

4. If $m>1$ and at least one of random varables $X_{1}, \ldots$, $X_{m}$ is constant, then $\left\langle X_{1}, \ldots, X_{m}\right\rangle=0$.

Proof can be found in (Malyshev and Minlos, 1991).

The following is a well-known lemma about semiinvariants of normal distribution.

\section{Lemma 2.2}

Suppose random variables $Y_{1}, Y_{2}, \ldots, Y_{m}$ have an independent multivariate normal distribution and $M=$ $\{1,2, \ldots, m\}$ is the set of their indices.

$$
\text { 1. If } k \geq 3 \text { and } i_{1}, \ldots, i_{k} \in M \text {, then }\left\langle Y_{i_{1}}, \ldots, Y_{i_{k}}\right\rangle=0 \text {. }
$$$$
\text { 2. If } i, j \in M \text { and } i \neq j \text {, then }\left\langle Y_{i}, Y_{j}\right\rangle=0 \text {. }
$$ 


\section{Definition 2.2}

We say that random variables $Y_{1}, \ldots, Y_{m}$ satisfy the $\boldsymbol{m}$ variate Carleman condition if:

$$
\sum_{n=1}^{\infty}\left(M_{2 n}\right)^{-\frac{1}{2 n}}=\infty, \text { where } M_{k}=\sum_{i=1}^{m}\left\langle Y_{i}^{k}\right\rangle
$$

For example, if $X$ is bounded, then it satisfies Carleman condition. The logarithmic normal distribution does not satisfy Carleman condition and is not defined uniquely by its moments. In order to prove that random variables $X_{1}, X_{2}, \ldots, X_{m}$ with identical distribution satisfy the $m$-variate Carleman condition, it is sufficient to check the 1-variate Carleman condition for only one of the random variables.

We will use the following version of Carleman theorem.

\section{Theorem 2.1. (Carleman theorem)}

Suppose random vectors $\left(X_{1}, \ldots, X_{m}\right)$ and $\left(Y_{1}, \ldots, Y_{m}\right)$ have equal corresponding moments and the variables $Y_{1}, \ldots, Y_{m}$ satisfy the $m$-variate Carleman condition. Then the random vectors $\left(X_{1}, \ldots, X_{m}\right)$ and $\left(Y_{1}, \ldots, Y_{m}\right)$ have the same probability distribution.

\section{Lemma 2.3}

If a random variable $Z$ has the standard normal distribution, then it satisfies the 1-variate Carleman condition.

\section{Proof}

Clearly, $\left\langle Z^{2 k}\right\rangle=(2 k-1)$ !! If integer $k \geq 1$, then $(2 k-1) ! ! \leq k^{k}$. Therefore

$$
\sum_{k=1}^{\infty}\left\langle Z^{2 k}\right\rangle^{-\frac{1}{2 k}} \geq \sum_{k=1}^{\infty} \frac{1}{\sqrt{k}}=\infty .
$$

Similarly it can be proven that a random variable with exponential distribution satisfies the 1-variate Carleman condition.

\section{Lemma 2.4}

Suppose random variables $X_{1}, X_{2}, \ldots, X_{m}$ are uncorrelated, identically distributed and satisfy the following condition:

$$
\left\langle X_{i_{1}}, \ldots, X_{i_{m}}\right\rangle=0 \text { for } k \geq 3 ; 1 \leq i_{1}, \ldots, i_{m} \leq m .
$$

Then $X_{1}, X_{2}, \ldots, X_{m}$ are independent and have a multivariate normal distribution.

\section{Proof}

Denote $\mu=\left\langle X_{i}\right\rangle, \sigma^{2}=\left\langle X_{i}, X_{i}\right\rangle$. Consider independent random variables $Z_{1}, Z_{2}, \ldots, Z_{m}$, where each $Z_{i}$ has the standard normal distribution and denote $Y_{i}=\sigma Z_{i}$. Then $Y_{1}, \ldots, Y_{m}$ are independent and have a multivariate normal distribution.

By Lemma 2.3 each $Z_{i}$ satisfies the 1-variate Carleman condition and so does each $Y_{i}$. Since $Y_{1}, \ldots, Y_{m}$ are identically distributed, they satisfy the $m$-variate Carleman condition.

By Lemma 2.2, the random vectors $\left(Y_{1}, \ldots, Y_{m}\right)$ and $\left(X_{1}-\mu, \ldots, X_{m}-\mu\right)$ have the same corresponding semiinvariants and the same corresponding moments, since semi-invariants uniquely determine moments. So by Carleman theorem, the random vectors $\left(Y_{1}, \ldots, Y_{m}\right)$ and $\left(X_{1}-\mu, \ldots, X_{m}-\mu\right)$ have the same distribution, which is an independent multivariate normal distribution. Hence the random vector $\left(X_{1}, \ldots, X_{m}\right)$ has an independent multivariate normal distribution.

\subsection{Interaction Model in Statistical Mechanics}

For the rest of the paper we fix a natural number $v \geq 1$ and consider a $v$-dimensional integer lattice:

$$
\mathbb{Z}^{v}=\left\{\left(t_{1}, \ldots, t_{v}\right) \mid t_{i} \in \mathbb{Z}, i=1,2, \ldots, v\right\}
$$

with the distance between any two points $s$ and $t$ defined by:

$$
\|s-t\|=\sum_{i=1}^{v}\left|s_{i}-t_{i}\right|
$$

Denote $\overline{0}=(0, \ldots, 0)$, the origin. Fix a set $D \subseteq \mathbb{R}$ with at least 2 elements and denote $\Omega=\left\{\omega \mid \omega: \mathbb{Z}^{v} \rightarrow D\right\}$. An element $\omega$ of $\Omega$ is called a configuration and is interpreted as a state of a physical system in statistical mechanics.

For each $t \in \mathbb{Z}^{v}$ a function $X_{t}: \Omega \rightarrow D$ is defined by the following:

$$
X_{t}(\omega)=\omega(t)
$$

We define $\Sigma$ as the $\sigma$-algebra generated by sets of the form $\{\omega \in \Omega \mid \omega(t) \leq a\}$ for all $t \in \mathbb{Z}^{v}$ and $a \in D$. We fix a probability measure $P_{0}$ on $(\Omega, \Sigma)$ such that:

for any $a \in D, P_{0}(\omega(t)<a)$ does not depend on $t$ and

for any $a_{1}, \ldots, a_{n} \in D$ and distinct $t_{1}, \ldots, t_{n} \in \mathbb{Z}^{v}$ :

$$
\begin{aligned}
& P_{0}\left(\omega\left(t_{1}\right)<a_{1}, \ldots, \omega\left(t_{n}\right)<a_{n}\right) \\
= & P_{0}\left(\omega\left(t_{1}\right)<a_{1}\right) \cdot \ldots \cdot P_{0}\left(\omega\left(t_{n}\right)<a_{n}\right) .
\end{aligned}
$$

Then $\left\{X_{t} \mid t \in \mathbb{Z}^{v}\right\}$ is an independent random field on the probability space $\left(\Omega, \Sigma, P_{0}\right)$ and this field is translation invariant. Clearly, the random variables $X_{t}$, $t \in \mathbb{Z}^{v}$, are identically distributed with respect to the measure $P_{0}$. 
We also assume that the following conditions are satisfied:

each $X_{t}$ has a finite moment of $m^{\text {th }}$ order, $m=1,2,3, \ldots$;

each $X_{t}$ satisfies the 1-variate Carleman condition:

$\sum_{n=1}^{\infty}\left(\left\langle X_{t}^{2 k}\right\rangle_{0}\right)^{-\frac{1}{2 k}}=\infty$

We denote $\langle\cdot\rangle_{0}$ the expectation with respect to the measure $P_{0}$.

\section{Note 1}

There always exists a probability measure $P_{0}$ satisfying (2) - (3) and for which $\left\{X_{t} \mid t \in \mathbb{Z}^{v}\right\}$ satisfies the conditions (4) - (5). Here is an example. Let $F(x)$ be a probability distribution function satisfying Carleman condition, that is:

$$
\sum_{n=1}^{\infty}\left(M_{2 n}\right)^{-\frac{1}{2 n}}=\infty, \text { where } M_{k}=\int_{-\infty}^{+\infty} x^{k} d F(x)
$$

As mentioned before, the normal and exponential distributions are some of the distributions satisfying Carleman condition.

Probability measure $P_{0}$ is defined by:

$$
P_{0}(\omega(t)<a)=F(a) \text { and equality (3). }
$$

Then the conditions (2) - (5) are satisfied.

We fix an increasing sequence $\Lambda_{N}$ of finite subsets of $\mathbb{Z}^{v}$ such that $\Lambda_{N} \subset \Lambda_{N+1}$ for any $N \in \mathbb{N}$ and

$$
\bigcup_{N=1}^{\infty} \Lambda_{N}=\mathbb{Z}^{v}
$$

Denote $R=\left\{\{s, t\} \mid s, t \in \mathbb{Z}^{v}\right.$ and $\left.\left\|s^{-} t\right\|=1\right\} . R$ is the set of all pairs of neighbouring nodes in the lattice $\mathbb{Z}^{v}$. Denote $R B=\left\{u \in \mathbb{Z}^{v} \mid\right.$ one coordinate of $u$ is 1 and the others are 0$\}. R B$ is the standard basis in $\mathbb{R}^{v}$.

\section{Definition 2.3}

Interaction model is defined by a triple of objects $(N, \lambda, \varphi)$, where

(i) $N \in \mathbb{N}, N \geq 1$;

(ii) $\lambda \in \mathbb{R}$;

(iii) for any $u \in R B, \varphi_{u}: \mathbb{R}^{2} \rightarrow \mathbb{R}$ is a Borel function such that $\left|\varphi_{u}\right| \leq 1$.

The interaction model includes a set $\Lambda_{N}$ (as defined before), potential $\Phi$ and interaction energy $U_{N}$ defined as follows.
1) For any $B \in R$ we define a random variable $\Phi_{B}$ on the probability space $\left(\Omega, \Sigma, P_{0}\right)$. Any $B \in R$ has the form $B=\{r, r+u\}$, where $u \in R B$, so we define:

$$
\Phi_{B}(\omega)=\varphi_{u}\left(X_{r}(\omega), X_{r+u}(\omega)\right)
$$

Such $\Phi_{B}$ represents interaction between neighbours $r$ and $r+u$.

2) Function $U_{N}: \Omega \rightarrow R$ is defined by the following:

$U_{N}(\omega)=-\lambda \sum_{B \in R, B \subset \Lambda_{N}} \Phi_{B}(\omega)$

$U_{N}(\omega)$ characterizes the energy of configuration $\omega$ in $\Lambda_{N}$.

This completes the definition of the interaction model.

Note 2

In the interaction model a union of the random fields $\left\{X_{t} \mid t \in \mathbb{Z}^{v}\right\}$ and $\left\{\Phi_{B} \mid B \in R\right\}$ is translation invariant. This means: for any $t_{1}, \ldots, t_{m}, r \in \mathbb{Z}^{v}$ and any $B_{1}, \ldots, B_{n} \in R$ the random vectors $\left(X_{t_{1}}, \ldots, X_{t_{m}}, \Phi_{B_{1}}, \ldots, \Phi_{B_{n}}\right) \quad$ and $\left(X_{t_{1}+r}, \ldots, X_{t_{m}+r}, \Phi_{B_{1}+\bar{r}}, \ldots, \Phi_{B_{n}+\bar{r}}\right)$ have the same distribution (here for $B_{i}=\left\{s_{i}, t_{i}\right\}, B_{i}+\bar{r}$ denotes $\left\{s_{i}+r, t_{i}+r\right\}$ ).

The interaction model describes a physical system with many particles represented by points of the set $\Lambda_{N}$ in the integer lattice. The random field $X_{t}$ describes some property of the physical system. The function $U_{N}$ characterizes the interaction energy of the system and $|\lambda|$ is proportional to the inverse temperature of the system. The parameter $\lambda$ also characterizes the strength of interaction between particles and we assume that only neighbouring particles interact.

\section{Example 2.2}

The statistical model with $\lambda=0$ describes a physical system with no interaction between its elements, e.g., ideal gas.

\section{Example 2.3}

Potts model with parameter $q(q \in \mathbb{N}, q \geq 1)$.

It is a particular case of interaction model, where $D=\{1,2, \ldots, q\}$ and the probability measure $P_{0}$ is defined by:

$$
P_{0}(\omega(t)=i)=\frac{1}{q}, i=1,2, \ldots, q, \text { and equality (3). }
$$


Since $D$ is finite, each $X_{t}$ satisfies the Carleman condition. The rest of the model is defined by:

$$
\begin{aligned}
& \varphi_{u}(x, y)=\delta(x, y), \text { where } \delta(x, y)= \begin{cases}1 & \text { if } x=y, \\
0 & \text { if } x \neq y .\end{cases} \\
& \text { So } \Phi_{\{s, t\}}(\omega)=\delta\left(X_{s}(\omega), X_{t}(\omega)\right) \text { and } \\
& U_{N}(\omega)=-\lambda \sum_{\{s, t\} \in R ; s, t \in \Lambda_{N}} \delta\left(X_{s}(\omega), X_{t}(\omega)\right)
\end{aligned}
$$

\subsection{Gibbs Modification}

Gibbs modification was introduced in (Malyshev and Minlos, 1991). Since it is important for our paper, we also provide the definition.

\section{Definition 2.4}

For the interaction model given by $(N, \lambda, \varphi)$ we define the associated probability space $\left(\Omega, \Sigma_{N}, P_{\lambda, N}\right)$ as follows:

1. The sample space is the set $\Omega$ as defined before.

2. $\Sigma_{N}$ is the sigma-algebra generated by $X_{t}, t \in \Lambda_{N}$.

3. For any event $A \in \Sigma_{N}$ the probability is defined by:

$$
P_{\lambda, N}(A)=\frac{\left\langle I_{A} e^{-U_{N}}\right\rangle_{0}}{\left\langle e^{-U_{N}}\right\rangle_{0}}
$$

where $I_{A}$ denotes the indicator of event $A$.

The probability measure $P_{\lambda, N}$ is called (finite) Gibbs modification on $\Lambda_{N}$. This completes the definition.

$$
\text { Note: Clearly, } P_{\lambda, N}(\Omega)=\frac{\left\langle 1 \cdot e^{-U_{N}}\right\rangle_{0}}{\left\langle e^{-U_{N}}\right\rangle_{0}}=1 \text {. }
$$

\section{Example 2.4}

Ising model with parameters $N, \lambda$ and $h$ is usually defined with probabilities:

$$
P(\omega(t)=1)=P(\omega(t)=-1)=\frac{1}{2}
$$

and potential $W_{N}=U_{N}+V_{N}$, where

$$
U_{N}=-\lambda \sum_{\{s, t\} \in R, s, t \in \Lambda_{N}} X_{s}(\omega) X_{t}(\omega) \text { and } V_{N}=-\lambda \sum_{s \in \Lambda_{N}} X_{s}(\omega)
$$

$\Sigma_{N}$ is defined in the same way as in the interaction model and probability measure $P_{G}$ is defined by:

$$
P_{G}(A)=\frac{\left\langle I_{A} e^{-W_{N}}\right\rangle_{0}}{\left\langle e^{-W_{N}}\right\rangle_{0}} \text { for any } A \in \Sigma_{N},
$$

where the expectations are with respect to the probability measure $P$.

We can define the Ising model as a particular case of interaction model. We take $D=\{-1,1\} ; P_{0}$ is defined by:

$$
\begin{aligned}
& P_{0}(\omega(t)=a)=\frac{e^{-h a}}{e^{-h}+e^{h}} \quad(a= \pm 1) \text { and equality (3); } \\
& \varphi_{u}(x, y)=x y .
\end{aligned}
$$

$$
\begin{aligned}
\text { So } \Phi_{\{s, t\}}(\omega)=X_{s}(\omega) X_{t}(\omega) \text { and } \\
\qquad U_{N}=-\lambda \sum_{\{s, t\} \in R, s, t \in \Lambda_{N}} X_{s}(\omega) X_{t}(\omega) .
\end{aligned}
$$

Then we show that the probability space $\left(\Omega, \Sigma_{\mathrm{N}}, P_{\lambda, N}\right)$ is the same as $\left(\Omega, \Sigma_{N}, P_{G}\right)$ in the usual definition of the Ising model.

Proof that $P_{G}=P_{\lambda, N}$.

Fix $A \in \Sigma_{N}$.

$$
\begin{aligned}
& \left\langle I_{A} e^{-W_{N}}\right\rangle=\sum_{\omega \in \Omega} I_{A}(\omega) e^{-W_{N}(\omega)} P(\omega) \\
& =\sum_{\omega \in A} e^{-U_{N}(\omega)-V_{N}(\omega)} \frac{1}{2^{\left|\Lambda_{N}\right|}} \\
& =\frac{1}{2^{\left|\Lambda_{N}\right|}} \sum_{\omega \in A} e^{-U_{N}(\omega)} e^{-h \sum_{s \in \Lambda_{N}} X_{s}(\omega)} \\
& =\frac{1}{2^{\left|\Lambda_{N}\right|}} \sum_{\omega \in A}\left(e^{-U_{N}} \prod_{s \in \Lambda_{N}} e^{-h X_{s}(\omega)}\right) \\
& =\left(\frac{1}{2}\right)^{\left|\Lambda_{N}\right|}\left(\frac{e^{-h}+e^{h}}{e^{-h}+e^{h}}\right)^{\left|\Lambda_{N}\right|} \sum_{\omega \in A}\left(e^{-U_{N}(\omega)} \prod_{s \in \Lambda_{N}} e^{-h \omega(s)}\right) \\
& =\left(\frac{e^{-h}+e^{h}}{2}\right)^{\left|\Lambda_{N}\right|} \sum_{\omega \in A}\left(e^{-U_{N}(\omega)} \prod_{s \in \Lambda_{N}} \frac{e^{-h \omega(s)}}{e^{-h}+e^{h}}\right) \\
& =\left(\frac{e^{-h}+e^{h}}{2}\right)^{\left|\Lambda_{N}\right|} \sum_{\omega \in A} e^{-U_{N}(\omega)} P_{0}(\omega) \\
& =\left(\frac{e^{-h}+e^{h}}{2}\right)^{\left|\Lambda_{N}\right|}\left\langle I_{A} e^{-U_{N}}\right\rangle_{0}
\end{aligned}
$$

Similarly, $\left\langle e^{-W_{N}}\right\rangle=\left(\frac{e^{-h}+e^{h}}{2}\right)^{\left|\Lambda_{N}\right|}\left\langle e^{-U_{N}}\right\rangle_{0}$.

Therefore

$$
P_{G}(A)=\frac{\left\langle I_{A} e^{-W_{N}}\right\rangle}{\left\langle e^{-W_{N}}\right\rangle}=\frac{\left\langle I_{A} e^{-U_{N}}\right\rangle_{0}}{\left\langle e^{-U_{N}}\right\rangle_{0}}=P_{\lambda, N}(A) .
$$

\section{Lemma 2.5}

Suppose $\lambda=0$. Then the following hold. 1. $P_{0, N}=P_{0}$ on $\Sigma_{N}$. 
2. $\left\{X_{t} \mid t \in \Lambda_{N}\right\}$ is an independent random field with respect to $P_{0, N}$.

3. The distribution of the random field $\left\{X_{t} \mid t \in \Lambda_{N}\right\}$ with respect to Gibbs modification $P_{0, N}$ does not depend on $N$.

4. Suppose $T=\left\{t_{1}, \ldots, t_{n}\right\} \subset \Lambda_{N}, S=\left\{s_{1}, \ldots, s_{m}\right\} \subset \Lambda_{N}$ and $T \cap S=\varnothing$. Suppose $F_{i}(i=1, \ldots, k)$ are random variables dependent on $X_{t_{1}}, \ldots, X_{t_{n}}$ and $G_{j}(j=1, \ldots, p)$ are random variables dependent on $X_{s_{1}}, \ldots, X_{s_{m}}$. Then the random vectors $\left(F_{1}, \ldots, F_{k}\right)$ and $\left(G_{1}, \ldots, G_{p}\right)$ are independent of each other with respect to $P_{0}$.

\section{Proof}

Let $\lambda=0$. Then $U_{N}=0$.

1. For any $A \in \Sigma_{N}, P_{0, N}(A)=\frac{\left\langle I_{A} e^{0}\right\rangle_{0}}{\left\langle e^{0}\right\rangle_{0}}=\left\langle I_{A}\right\rangle_{0}=P_{0}(A)$.

2. This part holds because the random variables $X_{t}$, $t \in \Lambda_{N}$, are independent with respect to the measure $P_{0}$.

3. Follows from part 1.

4. Follows from part 2.

\subsection{Gibbs Measure and Thermodynamic Limit}

The definition of Gibbs measure is given in (Dobrushin, 1968); it is a probability measure on $(\Omega$, $\Sigma)$. For our results it is sufficient to consider Gibbs measure $P_{\lambda}$ as the limit of Gibbs modifications $P_{\lambda, N}$ as $N \rightarrow \infty:$

$$
P_{\lambda}(A)=\lim _{N \rightarrow \infty} P_{\lambda, N}(A) \text { for any } A \in \Sigma
$$

Malyshev and Minlos (1991) established necessary and sufficient conditions when the equality (7) holds; the results in (Kashapov, 1980) imply that the equality (7) holds for all $\lambda$ with $|\lambda|<C$, where $C$ is the constant from our main theorem (Theorem 3.1).

Let us see what happens to the interaction model and associated probability space when $N \rightarrow \infty$. Clearly, the finite set $\Lambda_{N}$ transforms into the lattice $\mathbb{Z}^{v}, \Sigma_{N}$ transforms into $\Sigma$ and the Gibbs modification $P_{\lambda, N}$ transforms into the Gibbs measure $P_{\lambda}$.

\section{Definition 2.5}

The thermodynamic or macroscopic limit of interaction model is the lattice $\mathbb{Z}^{v}$ together with the limiting probability space $\left(\Omega, \Sigma, P_{\lambda}\right)$.

Clearly, $\left\{X_{t} \mid t \in \mathbb{Z}^{v}\right\}$ is a random field on the limiting probability space. We denote $\langle\cdot, \ldots, \cdot\rangle_{\lambda}$ semiinvariants with respect to the Gibbs measure $P_{\lambda}$.

\subsection{Renormalization Group}

The following concept was introduced by Kadanoff (1966).

\section{Definition 2.6}

Fix a natural number $k>1$ and a real number $\alpha \geq v$.

For each $r=\left(r_{1}, r_{2}, \ldots, r_{v}\right) \in \mathbb{Z}^{v}$ consider a cube $C_{r}^{k}$ of side length $k$ with vertex $k r$ :

$C_{r}^{k}=\left\{t \in \mathbb{Z}^{v} \mid k r_{i} \leq t_{i}<k\left(r_{i}+1\right), i=1,2, \ldots, v\right\}$.

A renormalization group $(\mathrm{RG})$ with parameters $k$ and $\alpha$ is a transformation that assigns to each random field $\left\{Z_{t}\right.$ $\left.\mid t \in \mathbb{Z}^{v}\right\}$ a new random field $\left\{Y_{r}^{(k)} \mid r \in \mathbb{Z}^{v}\right\}$ given by:

$$
Y_{r}^{(k)}=k^{-\frac{\alpha}{2}} \sum_{t \in C_{r}^{k}}\left(Z_{t}-\left\langle Z_{t}\right\rangle_{\lambda}\right)
$$

RG is a scaling transformation. It allows to study a physical system at different distance scales, such as atomic and molecular levels. Details of its physical interpretation can be found in (Kadanoff, 2013).

We are interested in the distribution of the result $Y_{r}^{(k)}$ of the RG transformation of the field $\left\{X_{t} \mid t \in \mathbb{Z}^{v}\right\}$.

\section{The Central Limit Theorem for the Field $X_{t}$ Transformed by RG}

\section{Theorem 3.1. (Main Theorem)}

Consider the thermodynamic limit of interaction model with parameter $\lambda$. Suppose a renormalization group with parameters $k$ and $\alpha$ transforms the random field $\left\{X_{t} \mid t \in \mathbb{Z}^{v}\right\}$ into a random field $\left\{Y_{i}^{(k)} \mid r \in \mathbb{Z}^{v}\right\}$.

There exists a positive constant $C$ such that for any $|\lambda|<C$ the following hold.

1. Suppose $\alpha>v$. Then the field $Y_{r}^{(k)} \rightarrow 0$ in mean square as $k \rightarrow \infty$.

2. Suppose $\alpha=v$. Then as $k \rightarrow \infty$, the random field $\left\{Y_{i}^{(k)} \mid r \in \mathbb{Z}^{v}\right\}$ converges in distribution to an independent random field with Gaussian distribution (i.e., any finite subset of the field has a multivariate normal distribution). Each of the variables of the limiting field has 0 expectation and the positive variance given by:

$$
V=\sum_{n=0}^{\infty} \lambda^{n} V_{n}
$$

where each coefficient $V_{n}$ is a finite sum of semiinvariants of $X_{t}$ and $\Phi_{B}$ with respect to $P_{0}\left(t \in \mathbb{Z}^{v}\right.$, $B \in R)$. Exact formula for coefficients $V_{n}$ is formula (27) in subsection 5.4. 
Proof is given in section 5 .

This theorem can be considered as a generalization of the classical Central Limit Theorem (CLT). Instead of a sequence of independent random variables we have a weakly dependent random field $\left\{X_{t} \mid t \in \mathbb{Z}^{v}\right\}$. It is weakly dependent because $|\lambda|$ is small and $\lambda$ characterizes the strength of interaction.

The classical CLT considers a sequence of independent identically distributed random variables with finite variances and states that their normalized sums converge in distribution to a normal random variable. Theorem 3.1.2) also states convergence in distribution and that the limiting distribution is normal but in this case it is the distribution of an independent normal field.

In other words, Theorem 3.1 states: in systems with weak interaction the distribution of the normalized sums over big regions is approximately independent and normal.

\section{Estimation of Dependencies}

\subsection{Estimation Theorem}

The proof of the main theorem in Section 5 is based on estimations of semi-invariants. In this section we prove an inequality (Theorem 4.1), which will be applied to estimating semi-invariants. This was inspired by Estimates of Intersection Number in (Malyshev and Minlos, 1991). Here we have improved our estimate from (Kachapova and Kachapov, 2015) and simplified the proof.

In this section we consider a countable set $\mathfrak{A}$ with a reflexive, symmetric binary relation. If elements $a, b$ of $\mathfrak{A}$ are in this relation, we say that $a$ and $b$ are linked. Thus, any element of $\mathfrak{A}$ is always linked to itself (reflexivity). If $a$ is linked to $b$, then $b$ is linked to $a$ (symmetry).

Denote $l(a)$ the number of elements in $\mathfrak{U}$ that are linked to $a$. In this section we assume that there is a constant $L$ such that $l(a) \leq L$ for all $a \in \mathfrak{U}$.

\section{Definition 4.1}

For any sequence $\alpha=\left(a_{1}, \ldots, a_{m}\right)$ of elements of $\mathfrak{U}$ we use the following notations:

1. $J(\alpha)=\left\{i_{1}, \ldots, i_{k}\right\}$ is the set of indexes of elements of $\alpha$ when the elements are written without repetition.

2. $n_{i}(\alpha)$ is the number of elements of $\alpha$ that equal $a_{i}$ $(i=1, \ldots, m)$. This number is called the multiplicity of $a_{i}$.

3. $v_{i}(\alpha)$ is the number of elements of $\alpha$ that are linked to $a_{i}(i=1, \ldots, m)$.

\section{Theorem 4.1. (Estimation Theorem)}

For any sequence $\alpha$ of elements of $\mathfrak{A}$ :

$$
\frac{1}{|\alpha|} \sum_{j \in J(\alpha)} n_{j} \ln \left(\frac{v_{j}}{n_{j}}\right) \leq \ln L,
$$

where $|\alpha|=m$ is the length of the sequence $\alpha, n_{j}=n_{j}(\alpha)$ and $v_{j}=v_{j}(\alpha)$.

This estimate cannot be improved.

\section{Proof}

Fix a sequence $\alpha=\left(a_{1}, \ldots, a_{m}\right)$ of elements of $\mathfrak{A}$. Denote $J=J(\alpha)$ for brevity. We define a link matrix $d_{i j}$ as follows:

$$
d_{i j}=\left\{\begin{array}{l}
1 \text { if } a_{i} \text { is linked to } a_{j}, \\
0 \text { otherwise. }
\end{array}\right.
$$

Then for any $j=1, \ldots, m$ :

$$
\begin{gathered}
\sum_{i \in J} d_{i j} \leq l\left(a_{j}\right) \leq L \text { and } \\
\sum_{i \in J} v_{i}=\sum_{i \in J} \sum_{j \in J} d_{i j} n_{j}=\sum_{j \in J} n_{j} \sum_{i \in J} d_{i j} \leq L \sum_{j \in J} n_{j} .
\end{gathered}
$$

Thus,

$$
\sum_{i \in J} v_{i} \leq m L
$$

Next we use Jensen inequality, which states for a concave function $f$ and numbers $x_{1}, \ldots, x_{n}$ in its domain:

$$
\frac{1}{n} \sum_{i=1}^{n} f\left(x_{i}\right) \leq f\left(\frac{1}{n} \sum_{i=1}^{n} x_{i}\right) .
$$

We apply the Jensen inequality to the concave function logarithm and $x_{i}=\frac{v_{i}}{n_{i}} \quad(i=1, \ldots, m)$ :

$$
\begin{gathered}
\frac{1}{m} \sum_{j \in J} n_{j} \ln \left(\frac{v_{j}}{n_{j}}\right)=\frac{1}{m} \sum_{i=1}^{m} \ln \left(\frac{v_{i}}{n_{i}}\right) \\
\leq \ln \left(\frac{1}{m} \sum_{i=1}^{m} \frac{v_{i}}{n_{i}}\right) \leq \ln \left(\frac{1}{m} \sum_{j \in J} n_{j} \frac{v_{j}}{n_{j}}\right) \\
=\ln \left(\frac{1}{m} \sum_{j \in J} v_{j}\right) \leq \ln \left(\frac{1}{m} m L\right)=\ln L \text { by (9). }
\end{gathered}
$$

The following example shows that the estimate cannot be improved. $\mathfrak{A}$ is an arbitrary countable set and $m$ is any positive integer. We take $\alpha=\left(a_{1}, \ldots, a_{m}\right)$, where all $a_{i}$ are distinct. We assume that any $a_{i}$ and $a_{j}$ are linked $(i, j=1, \ldots, m)$ and there are no other links in $\mathfrak{A}$. Then for each $i=1, \ldots, m, n_{i}(\alpha)=1, v_{i}(\alpha)=l\left(a_{i}\right)=m$ and $L=m$. The left-hand side of $(8)$ is:

$$
\frac{1}{m} \sum_{j \in J} 1 \cdot \ln m=\frac{1}{m} \cdot m \cdot \ln m=\ln m=\ln L .
$$




\subsection{Application of the Estimation Theorem to Semi-Invariants}

First we introduce some notations. We take $\mathfrak{A}=$ $R \cup\left\{\{t\} \mid t \in \mathbb{Z}^{v}\right\}$. Two subsets $T$ and $S$ of $\mathbb{Z}^{v}$ are said to be linked if $T \cap S \neq \varnothing$. By Lemma 2.5.4), if sets $T$ and $S$ are not linked, then they correspond to independent random vectors. So links correspond to possible dependencies of random vectors.

Any element of the form $\{t\}$ is linked to itself and to $2 v$ elements of the form $\{t, r\}$, so $l(\{t\})=2 v+1$. Any element of the form $\{r, s\}$ is linked to elements $\{r\},\{s\}$, $2 v$ elements of the form $\{r, t\}$ and $2 v$ elements of the form $\{s, t\}$, so the total is $4 v+1$ (because the element $\{r$, $s\}$ is counted twice). Then $L=4 v+1$ (each element of $\mathfrak{A}$ is linked to at most $4 v+1$ elements).

\section{Definition 4.2} pairs:

1. A family (of elements of the set $\mathfrak{U}$ ) is a set of

$$
\mathcal{A}=\left\{\left(T_{1}, n_{1}\right), \ldots,\left(T_{m}, n_{m}\right)\right\}
$$

where $T_{1}, \ldots, T_{m}$ are distinct elements of $\mathfrak{A}$ and $n_{i} \geq 1$ for each $i=1, \ldots, m$.

2. The number $n_{i}$ is called the multiplicity of element $T_{i}$ in the family $\mathcal{A}$.

3. We denote the length of the family $\mathcal{A}$ as

$$
\begin{gathered}
|\mathcal{A}|=n_{1}+n_{2}+\ldots+n_{m} \\
\text { and } \mathcal{A} !=n_{1} ! \cdot n_{2} ! \cdot \ldots \cdot n_{m} !
\end{gathered}
$$

We use letters $\mathcal{A}, \Psi, \ldots$ for families. The same elements $T_{1}, \ldots, T_{n} \in \mathfrak{A}$ can be represented as a sequence or a family.

\section{Definition 4.3}

1. Any sequence $\alpha=\left(T_{1}, \ldots, T_{n}\right)$ reduces to a family:

$$
\left\{\left(\bar{T}_{1}, n_{1}\right), \ldots,\left(\bar{T}_{q}, n_{q}\right)\right\}
$$

where $\bar{T}_{1}, \ldots, \bar{T}_{q}$ are the elements $T_{1}, \ldots, T_{n}$ written without repetitions and each $n_{i}$ is the number of times that $\bar{T}_{i}$ is repeated in $\alpha ; n_{1}+\ldots+n_{q}=n$.

For each family $\mathcal{A}$ of length $n$ there are $n ! / \mathcal{A}$ ! sequences that reduce to $\mathcal{A}$.

2 . If a sequence $\alpha$ reduces to a family $\mathcal{A}$ we denote $\alpha !=\mathcal{A} !$

\section{Lemma 4.1}

Denote $M_{k}=\left\langle\left|X_{\overline{0}}\right|^{k}\right\rangle_{0}$ and

$$
C(m, X)=\max \left\{M_{k_{1}} \cdot \ldots \cdot M_{k_{l}}\right\}
$$

where the maximum is taken over all sequences of numbers $k_{1}, \ldots, k_{l}$ with $k_{1}+\ldots+k_{l}=m$.

Denote $C_{1}=3 e(4 v+1)$. For any sequence $\beta=\left(B_{1}, \ldots\right.$, $\left.B_{n}\right)$ of elements of $R$ and any sequence $\tau=\left(t_{1}, \ldots, t_{m}\right)$ of elements of $\mathbb{Z}^{v}$, the following holds:

$\left|\left\langle X_{\tau}^{\backslash}, \Phi_{\beta}^{\prime}\right\rangle_{0}\right| \leq C(|\tau|, X)\left(C_{1}\right)^{|\tau|+|\beta|} \tau ! \beta !$, where

$\left\langle X_{\tau}^{\backslash}, \Phi_{\beta}^{\backslash}\right\rangle_{0}=\left\langle X_{t_{1}}, \ldots, X_{t_{m}}, \Phi_{B_{1}}, \ldots, \Phi_{B_{n}}\right\rangle_{0}, \quad$ the semiinvariant with respect to $P_{0}$.

\section{Proof}

Consider a sequence $\alpha=\left(A_{1}, \ldots, A_{p}\right)$ of elements of $\mathfrak{A}$ and the set $I=\{1, \ldots, p\}$ of their indices. Consider random variables $F_{1}, \ldots, F_{p}$ such that each $F_{i}$ depends on $X_{t}, t \in A_{i}(i=1, \ldots, p)$.

Denote $F_{S}=\prod_{i \in S} F_{i}$ for any $S \subseteq I$ and denote

$$
D(\alpha)=\max \left|\left\langle F_{S_{1}}\right\rangle_{0} \cdot \ldots \cdot\left\langle F_{S_{l}}\right\rangle_{0}\right|
$$

where the maximum is taken over all partitions $\left\{S_{1}, \ldots\right.$, $\left.S_{l}\right\}$ of $I$. If each random variable $F_{i}(i=1, \ldots, p)$ satisfies Carleman condition, then $D(\alpha)<\infty$.

Suppose the sequence $\alpha$ reduces to a family $\mathcal{A}=$ $\left\{\left(\bar{A}_{1}, n_{1}\right), \ldots,\left(\bar{A}_{q}, n_{q}\right)\right\}$ and $J=J(\alpha)$. Theorem 1 on pg. 69 of (Malyshev and Minlos, 1991) implies that:

$\left|\left\langle F_{\alpha}\right\rangle_{0}\right| \leq \frac{3}{2} D(\alpha) \prod_{i \in I}\left(3 v_{i}\right), \quad$ where $\quad\left\langle F_{\alpha}^{\prime}\right\rangle_{0}=\left\langle F_{1}, \ldots, F_{p}\right\rangle_{0}$.

Then

$$
\left|\left\langle F_{\alpha}\right\rangle_{0}\right| \leq \frac{3}{2} D(\alpha) 3^{p} \prod_{j \in J}\left(v_{j}\right)^{n_{j}} .
$$

By the Estimation Theorem (Theorem 4.1) we have:

$$
\ln \left(\prod_{j \in J}\left(v_{j}\right)^{n_{j}}\right)=\sum_{j \in J} n_{j} \ln v_{j} \leq|\alpha| \ln L+\sum_{j \in J} n_{j} \ln n_{j},
$$

where $L=4 v+1$. So

$$
\prod_{j \in J}\left(v_{j}\right)^{n_{j}} \leq e^{|\alpha| \ln L} \cdot e^{\sum_{j \in J} n_{j} \ln n_{j}}=L^{|\alpha|} \prod_{j \in J}\left(n_{j}\right)^{n_{j}} .
$$

From Stirling formula we have $k^{k}<\frac{1}{2} k ! e^{k}$ for any positive integer $k$. Then by (10):

$$
\left|\left\langle F_{\alpha}\right\rangle_{0}\right| \leq \frac{3}{2} D(\alpha) 3^{|\alpha|} L^{|\alpha|} \prod_{j \in J}\left(n_{j}\right)^{n_{j}}
$$




$$
<D(\alpha)(3 L)^{|\alpha|} \prod_{j \in J} n_{j} ! e^{n_{j}}=D(\alpha)(3 e L)^{|\alpha|} \alpha ! .
$$

Thus,

$$
\left|\left\langle F_{\alpha}^{\dagger}\right\rangle_{0}\right| \leq D(\alpha)\left(C_{1}\right)^{|\alpha|} \alpha !
$$

Now we take $\alpha=\tau * \beta=\left(t_{1}, \ldots, t_{m}, B_{1}, \ldots, B_{n}\right)$, a concatenation of the sequences $\tau$ and $\beta$. We take $F_{i}=$ $X_{t_{i}}$ for $i=1, \ldots, m$ and $F_{m+j}=\Phi_{B_{j}}$ for $j=1, \ldots, n$. Then by (11):

$$
\left|\left\langle X_{\tau}^{\prime}, \Phi_{\beta}^{\prime}\right\rangle_{0}\right| \leq D(\alpha)\left(C_{1}\right)^{|\tau|+|\beta|} \tau ! \beta !
$$

Since each $\left|\Phi_{B_{j}}\right| \leq 1$, we have $D(\alpha)=D(\tau * \beta) \leq$ $\max \left\{\left\langle\left|X_{S_{1}}\right|\right\rangle_{0} \cdot \ldots \cdot\left\langle\left|X_{S_{l}}\right|\right\rangle_{0}\right\}$, where the maximum is taken over all partitions $\left\{S_{1}, \ldots, S_{l}\right\}$ of $\tau=\left(t_{1}, \ldots, t_{m}\right)$. For a fixed partition $\left\{S_{1}, \ldots, S_{l}\right\}$ denote $k_{i}=\left|S_{i}\right|, i=1, \ldots, l$; then $k_{1}+\ldots+k_{l}=m$.

From Hölder inequality we get by induction for any random variables $Y_{1}, \ldots, Y_{n}$ :

$$
\left|\left\langle Y_{1} \cdot \ldots \cdot Y_{n}\right\rangle_{0}\right| \leq\left(\left\langle\left|Y_{1}\right|^{n}\right\rangle_{0}\right)^{\frac{1}{n}} \cdot \ldots \cdot\left(\left\langle\left|Y_{n}\right|^{n}\right\rangle_{0}\right)^{\frac{1}{n}} .
$$

So each $\left|\left\langle X_{S_{i}}\right\rangle_{0}\right| \leq\left\langle\left|X_{\overline{0}}\right|^{k_{i}}\right\rangle_{0}=M_{k_{i}}$ because all $X_{t}$ have the same distribution. Therefore $\left|\left\langle X_{S_{i}}\right\rangle_{0} \cdot \ldots \cdot\left\langle X_{S_{l}}\right\rangle_{0}\right|$ $\leq M_{k_{1}} \cdot \ldots \cdot M_{k_{l}}$ and $D(\alpha) \leq C(m, X)$. Then by $(12)$ :

$$
\left|\left\langle X_{\tau}^{\lfloor}, \Phi_{\beta}^{\prime}\right\rangle_{0}\right| \leq C(m, X)\left(C_{1}\right)^{|\tau|+|\beta|} \tau ! \beta !
$$

\section{Proof of the Central Limit Theorem for the Interaction Model}

\subsection{Semi-Invariants with Respect to Gibbs Measure}

In this subsection we prove a series of lemmas about estimates and semi-invariants and later we use these lemmas to prove the main theorem.

\section{Definition 5.1}

Suppose $\Psi=\left\{\left(B_{1}, n_{1}\right), \ldots,\left(B_{k}, n_{k}\right)\right\}$ is a family of elements of $R$.

1. We define its associated graph $G(\Psi)$ as follows.

For each $i=1, \ldots, k, B_{i}$ has the form $B_{i}=\left\{r_{i}, s_{i}\right\}$. The points $r_{i}$ and $s_{i}$ belong to the set of vertices of $G(\Psi)$ and there are $n_{i}$ edges between $r_{i}$ and $s_{i}$. There are no other vertices or edges.

2. We say that the family $\Psi$ connects a sequence $\tau$ of elements of $\mathbb{Z}^{v}$ if the associated graph $G(\Psi)$ is connected and the set of its vertices contains all elements of the sequence $\tau$.

Thus, the associated graph has $|\Psi|=n_{1}+\ldots+n_{k}$ edges. The mapping $\Psi \mapsto G(\Psi)$ is a one-to-one mapping of families of elements of $R$ to this type of graphs on $\mathbb{Z}^{v}$.

A semi-invariant is a symmetrical functional, the order of random variables is not important. If a sequence $\beta$ of elements of $R$ reduces to a family $\Psi$, we denote $\left\langle X_{\tau}^{\backslash}, \Phi_{\Psi}^{\backslash}\right\rangle_{0}=\left\langle X_{\tau}^{\backslash}, \Phi_{\beta}^{\backslash}\right\rangle_{0}$.

\section{Lemma 5.1}

If a family $\Psi$ of elements of $R$ does not connect a sequence $\tau$ of elements of $\mathbb{Z}^{v}$, then $\left\langle X_{\tau}^{\downarrow}, \Phi_{\Psi}^{\backslash}\right\rangle_{0}=0$.

\section{Proof}

Denote $G=G(\Psi)$. Fix a sequence $\beta$ that reduces to the family $\Psi$. Suppose $\Psi$ does not connect $\tau$. There are two cases.

Case 1. Some elements of $\tau$ are not vertices of $G$.

Without loss of generality we can assume that exactly first $q$ elements of $\tau$ are not vertices of $G ; \tau=\left(t_{1}, \ldots, t_{q}\right.$, $\left.t_{q+1}, \ldots, t_{m}\right)$ and $\beta=\left(B_{1}, \ldots, B_{n}\right)$. Then by Lemma 2.5.4), the random vectors $\left(X_{t_{1}}, \ldots, X_{t_{q}}\right)$ and $\left(X_{t_{q+1}}, \ldots, X_{t_{m}}, \Phi_{B_{1}}, \ldots, \Phi_{B_{n}}\right)$ are independent of each other and by Lemma 2.1.2):

$$
\left\langle X_{\tau}^{\backslash}, \Phi_{\beta}^{\backslash}\right\rangle_{0}=\left\langle X_{t_{1}}, \ldots, X_{t_{q}}, X_{t_{q+1}}, \ldots, X_{t_{m}}, \Phi_{B_{1}}, \ldots, \Phi_{B_{n}}\right\rangle_{0}=0
$$

Case 2. All elements of $\tau$ are vertices of $G$ but $G$ is not connected.

Then $G=G_{1} \cup G_{2}$, where $G_{1}$ and $G_{2}$ are disjoint graphs. Without loss of generality we can assume: $\tau=$ $\left(t_{1}, \ldots, t_{q}, s_{1}, \ldots, s_{l}\right)$ and $\beta=\left(A_{1}, \ldots, A_{m}, B_{1}, \ldots, B_{n}\right)$, where $G_{1}$ contains $t_{1}, \ldots, t_{q}$ and all elements of $A_{1} \cup \ldots \cup A_{m}$ as vertices; $G_{2}$ contains $s_{1}, \ldots, s_{l}$ and all elements of $B_{1} \cup \ldots \cup$ $B_{n}$ as vertices. By Lemma 2.5.4), the random vectors $\left(X_{t_{1}}, \ldots, X_{t_{q}}, \Phi_{A_{1}}, \ldots, \Phi_{A_{m}}\right)$ and $\left(X_{s_{1}}, \ldots, X_{s_{l}}, \Phi_{B_{1}}, \ldots, \Phi_{B_{n}}\right)$ are independent of each other and by Lemma 2.1.2):

$$
\begin{aligned}
& \left\langle X_{\tau}^{\backslash}, \Phi_{\beta}^{\prime}\right\rangle_{0} \\
& =\left\langle X_{t_{1}}, \ldots, X_{t_{q}}, \Phi_{A_{1}}, \ldots, \Phi_{A_{m}}, X_{s_{1}}, \ldots, X_{s_{l}}, \Phi_{B_{1}}, \ldots, \Phi_{B_{n}}\right\rangle_{0}=0 .
\end{aligned}
$$


The following lemma is mentioned by several authors without a proof or with a complicated proof. Here we provide a short, simple proof giving an explicit value for the estimation constant.

\section{Lemma 5.2}

Denote $C_{2}=4 v^{2}$. Fix a sequence $\tau$ of points in $\mathbb{Z}^{v}$ and a natural number $n \geq 1$. The number of families $\Psi$ such that $|\Psi|=n$ and $\Psi$ connects $\tau$, is not greater than $\left(C_{2}\right)^{n}$.

\section{Proof}

For a family $\Psi=\left\{\left(B_{1}, n_{1}\right), \ldots,\left(B_{k}, n_{k}\right)\right\}$ consider the associated graph $G=G(\Psi)$. A new graph $G^{\prime}$ is obtained from $G$ by adding for every edge another edge with the same ends. So $G^{\prime}$ has $2 n$ edges. Each vertex of $G^{\prime}$ has an even degree and $G^{\prime}$ is connected, hence $G^{\prime}$ has an Eulerian trail, that is a closed path which includes every edge of the graph exactly once; the length of such a path is $2 n$.

Therefore the number of the families with $|\Psi|=n$ that connect $\tau$, is not greater than the number of paths with $2 n$ steps through $\tau$ going along edges of the lattice $\mathbb{Z}^{v}$. There are at most $2 v$ directions at each vertex. Therefore the number of such paths is not greater than $(2 v)^{2 n}=\left(C_{2}\right)^{n}$ for $C_{2}=(2 v)^{2}$.

For a sequence $\tau=\left(t_{1}, \ldots, t_{m}\right)$ denote $\left\langle X_{\tau}^{\prime}\right\rangle_{\lambda, N}$ $=\left\langle X_{t_{1}}, \ldots, X_{t_{m}}\right\rangle_{\lambda, N}$, the semi-invariant with respect to $P_{\lambda, N}$ and $\left\langle X_{\tau}^{\backslash}\right\rangle_{\lambda}=\left\langle X_{t_{1}}, \ldots, X_{t_{m}}\right\rangle_{\lambda}$, the semi-invariant with respect to $P_{\lambda}$. In the Definition 2.4 we expressed the measure $P_{\lambda, N}$ in terms of measure $P_{0}$. The following lemma describes a connection between semi-invariants with respect to these measures.

\section{Lemma 5.3}

Denote $C_{3}=\left(2 C_{1} C_{2}\right)^{-1}$, where $C_{1}$ is the constant from Lemma 4.1 and $C_{2}$ is the constant from Lemma 5.2. Fix $N>1$ and a sequence $\tau$ of points in $\Lambda_{N}$. The following equality holds:

$$
\left\langle X_{\tau}^{\backslash}\right\rangle_{\lambda, N}=\sum_{n=0}^{\infty} \lambda^{n} \sum_{\Psi} \frac{1}{\Psi !}\left\langle X_{\tau}^{\dagger}, \Phi_{\Psi}^{\backslash}\right\rangle_{0},
$$

where the finite inner sum is taken over all families $\Psi=$ $\left\{\left(B_{1}, n_{1}\right), \ldots,\left(B_{k}, n_{k}\right)\right\}$ such that $|\Psi|=n, \Psi$ connects $\tau$ and each $B_{i} \subset \Lambda_{N}$. The series (13) converges absolutely and uniformly for $\lambda \in\left[-C_{3}, C_{3}\right]$.

\section{Proof}

The semi-invariant with respect to Gibbs measure can be expanded in Taylor series:

$$
\begin{aligned}
& \left\langle X_{\tau}^{\dagger}\right\rangle_{\lambda, N}=\left\langle X_{t_{1}}, X_{t_{2}}, \ldots, X_{t_{m}}\right\rangle_{\lambda, N} \\
& =\sum_{n=0}^{\infty} \frac{(-1)^{n}}{n !}\langle X_{t_{1}}, X_{t_{2}}, \ldots, X_{t_{m}}, \underbrace{U_{N}, \ldots, U_{N}}_{n \text { times }}\rangle_{0},
\end{aligned}
$$

where $U_{N}=-\lambda \sum_{B \in R, B \subset \Lambda_{N}} \Phi_{B}$ is the potential of the interaction model.

The proof of (14) can be found in (Malyshev and Minlos, 1991), pg. 34. Expanding (14) we get:

$$
\begin{aligned}
& \left\langle X_{\tau}^{\dagger}\right\rangle_{\lambda, N} \\
& =\sum_{n=0}^{\infty} \frac{(-1)^{n}}{n !}\left\langle X_{t_{1}}, \ldots, X_{t_{m}},-\lambda \sum_{B_{1} \in R, B_{1} \subset \Lambda_{N}} \Phi_{B_{1}}, \ldots,-\lambda \sum_{B_{n} \in R, B_{n} \subset \Lambda_{N}} \Phi_{B_{n}}\right\rangle_{0} \\
& =\sum_{n=0}^{\infty} \frac{\lambda^{n}}{n !} \sum_{B_{1}, \ldots, B_{n} \in R, \text { each }}\left\langle X_{B_{i} \in \Lambda_{N}}\left\langle X_{t_{1}}, \ldots, X_{t_{m}}, \Phi_{B_{1}}, \ldots, \Phi_{B_{n}}\right\rangle_{0} .\right.
\end{aligned}
$$

So

$$
\left\langle X_{\tau}^{\backslash}\right\rangle_{\lambda, N}=\sum_{n=0}^{\infty} \lambda^{n} a_{N, n}
$$

where $a_{N, n}=\frac{1}{n !} \sum_{\beta}\left\langle X_{\tau}^{\dagger}, \Phi_{\beta}^{\prime}\right\rangle_{0}$.

The last sum is finite and is taken over all sequences $\beta=\left(B_{1}, B_{2}, \ldots, B_{n}\right)$ of elements of $R$ such that each $B_{i} \subset \Lambda_{N}$. In this sum we can take only the sequences $\beta$ that connect $\tau$ because for others the corresponding addends equal 0 by Lemma 5.1. If $n=$ 0 , then $a_{N, 0}=\left\langle X_{\tau}^{\dagger}\right\rangle_{0}$.

For each family $\Psi$ of length $n$ there are $\frac{n !}{\Psi !}$ sequences that reduce to $\Psi$. Therefore

$a_{N, n}=\sum_{\Psi} \frac{1}{\Psi !}\left\langle X_{\tau}^{\downarrow}, \Phi_{\Psi}^{\dagger}\right\rangle_{0}$,

where the sum is taken over all families $\Psi=$ $\left\{\left(B_{1}, n_{1}\right), \ldots,\left(B_{k}, n_{k}\right)\right\}$ such that $|\Psi|=n, \Psi$ connects $\tau$ and each $B_{i} \subset \Lambda_{N}$. So we have proven the equality (13).

It remains to prove that the series converges absolutely and uniformly on $\left[-C_{3}, C_{3}\right]$. By Lemma 4.1:

$$
\begin{aligned}
& \left|a_{N, n}\right| \leq \sum_{\Psi} \frac{1}{\Psi !}\left|\left\langle X_{\tau}^{\dagger}, \Phi_{\Psi}^{\backslash}\right\rangle_{0}\right| \\
& \leq \sum_{\Psi} \frac{1}{\Psi !} C(|\tau|, X)\left(C_{1}\right)^{|\tau|+n} \tau ! \Psi !=\sum_{\Psi}\left(C_{1}\right)^{n} q(\tau),
\end{aligned}
$$


where $q(\tau)=\left(C_{1}\right)^{|\tau|} \tau ! C(|\tau|, X)$ does not depend on $n$. By Lemma 5.2, the number of addends in the sum (16) is not greater than $\left(C_{2}\right)^{n}$. So $\left|a_{N, n}\right| \leq\left(C_{2}\right)^{n}\left(C_{1}\right)^{n} q(\tau)$ and for $\lambda \in\left[-C_{3}, C_{3}\right]:$

$$
\left|\lambda^{n} a_{N, n}\right| \leq\left(C_{3}\right)^{n}\left(C_{1} C_{2}\right)^{n} q(\tau)=\frac{1}{2^{n}} q(\tau)
$$

Therefore the series in (15) converges absolutely and uniformly on $\left[-C_{3}, C_{3}\right]$.

\section{Lemma 5.4}

Fix a sequence $\tau$ of points in $\mathbb{Z}^{v}$. For any $|\tau|<C_{3}$ (where $C_{3}$ is the constant from Lemma 5.3) the following equality holds:

$$
\left\langle X_{\tau}^{\backslash}\right\rangle_{\lambda}=\sum_{n=0}^{\infty} \lambda^{n} \sum_{\Psi} \frac{1}{\Psi !}\left\langle X_{\tau}^{\backslash}, \Phi_{\Psi}^{\backslash}\right\rangle_{0}
$$

where the finite inner sum is taken over all families $\Psi$ with $|\Psi|=n$ that connect $\tau$. The series (17) converges absolutely and uniformly for $\lambda \in\left[-C_{3}, C_{3}\right]$.

\section{Proof}

It is proven in (Malyshev and Minlos, 1991) that $\lim _{N \rightarrow \infty}\left\langle X_{\tau}^{\backslash}\right\rangle_{\lambda, N}=\left\langle X_{\tau}^{\backslash}\right\rangle_{\lambda}$. By taking the limit of both sides of (13) as $N \rightarrow \infty$ we get the equality (17). Similarly to Lemma 5.3 we estimate the common term of the series (17), which proves its absolute and uniform convergence.

\section{Lemma 5.5}

If $|\lambda|<C_{3}$ (where $C_{3}$ is the constant from Lemma 5.3 ), then any finite set of the random variables $X_{t}$, $t \in \mathbb{Z}^{v}$, satisfy the Carleman condition with respect to measure $P_{\lambda}$.

\section{Proof}

Fix $t \in \mathbb{Z}^{v}$ and denote $\tau=(t)$, a sequence of length 1 . Similarly to Lemma 5.4, we can show that for any natural $l$ :

$$
\left\langle X_{t}^{2 l}\right\rangle_{\lambda}=\sum_{n=0}^{\infty} \lambda^{n} \sum_{\Psi} \frac{1}{\Psi !}\left\langle X_{t}^{2 l}, \Phi_{\Psi}^{\lfloor}\right\rangle_{0},
$$

where the finite inner sum is taken over all families $\Psi$ with $|\Psi|=n$ that connect $\tau=(t)$.

Similarly to the proof of Lemma 5.3, we can estimate the coefficient for $\lambda^{n}$ :

$$
\left|\sum_{\Psi} \frac{1}{\Psi !}\left\langle X_{t}^{2 l}, \Phi_{\Psi}^{\backslash}\right\rangle_{0}\right| \leq\left(C_{2}\right)^{n}\left\langle X_{\overline{0}}^{2 l}\right\rangle_{0}\left(C_{1}\right)^{1+n},
$$

since $|\tau|=\tau !=1$. Then for $|\lambda|<C_{3}$ :

$$
\begin{gathered}
\left\langle X_{t}^{2 l}\right\rangle_{\lambda} \leq \sum_{n=0}^{\infty}|\lambda|^{n}\left\langle X_{\overline{0}}^{2 l}\right\rangle_{0} C_{1}\left(C_{1} C_{2}\right)^{n} \\
\leq \sum_{n=0}^{\infty} C_{1}\left\langle X_{\overline{0}}^{2 l}\right\rangle_{0}\left(C_{3} C_{1} C_{2}\right)^{n}=C_{1}\left\langle X_{\overline{0}}^{2 l}\right\rangle_{0} \sum_{n=0}^{\infty} \frac{1}{2^{n}} \\
\left\langle X_{t}^{2 l}\right\rangle_{\lambda} \leq 2 C_{1}\left\langle X_{\overline{0}}^{2 l}\right\rangle_{0} .
\end{gathered}
$$

Since $X_{\overline{0}}$ satisfies Carleman condition with respect to $P_{0}$ by conditions (4)-(5), then by (19) $X_{t}$ satisfies Carleman condition with respect to $P_{\lambda}$. Since all $X_{t}$ are identically distributed, then any finite set of them satisfy Carleman condition with respect to $P_{\lambda}$.

\subsection{Estimation of Semi-Invariants for $R G$}

In this subsection we estimate semi-invariants $\left\langle Y_{r_{1}}^{(k)}, Y_{r_{2}}^{(k)}, \ldots, Y_{r_{m}}^{(k)}\right\rangle_{\lambda}$, where each $Y_{r}^{(k)}$ is the result of RG transformation of the random field $\left\{X_{t} \mid t \in \mathbb{Z}^{v}\right\}$ :

$Y_{r}^{(k)}=k^{-\frac{\alpha}{2}} \sum_{t \in C_{r}^{k}}\left(X_{t}-\mu\right)$,

where $\left\langle X_{t}\right\rangle_{\lambda}=\mu$ does not depend on $t$.

\section{Lemma 5.6}

1. For any $r \in \mathbb{Z}^{v},\left\langle Y_{r}^{(k)}\right\rangle_{\lambda}=0$.

2. For $m>1$, any $r_{1}, \ldots, r_{m} \in \mathbb{Z}^{v}$ and $|\lambda|<C_{3}$ :

$$
\left\langle Y_{r_{1}}^{(k)}, Y_{r_{2}}^{(k)}, \ldots, Y_{r_{m}}^{(k)}\right\rangle_{\lambda}=k^{-m \alpha / 2} \sum_{n=0}^{\infty} \lambda^{n} \sum_{\tau} \sum_{\Psi} \frac{1}{\Psi !}\left\langle X_{\tau}^{\downarrow}, \Phi_{\Psi}^{\backslash}\right\rangle_{0},
$$

where the first inner sum is over all sequences $\tau=$ $\left(t_{1}, \ldots, t_{m}\right)$ with each $t_{i} \in C_{r_{i}}^{k}$ and the second inner sum is over all families $\Psi$ with $|\Psi|=n$ that connect $\tau$.

The series (21) converges absolutely and uniformly on $\left[-C_{3}, C_{3}\right]$.

\section{Proof}

1. Obvious

2. For $|\lambda|<C_{3}$ and any sequence $\tau=\left(t_{1}, \ldots, t_{m}\right)$ of elements of $\mathbb{Z}^{v}$ we have by Lemma 2.1.1) and 4):

$$
\left\langle X_{t_{1}}-\mu, \ldots, X_{t_{m}}-\mu\right\rangle_{\lambda}=\left\langle X_{t_{1}}, \ldots, X_{t_{m}}\right\rangle_{\lambda}=\left\langle X_{\tau}^{\backslash}\right\rangle_{\lambda} .
$$

So

$$
\begin{aligned}
& \left\langle Y_{r_{1}}^{(k)}, \ldots, Y_{r_{m}}^{(k)}\right\rangle_{\lambda}=k^{\left(-\frac{\alpha}{2}\right)^{m}} \sum_{\tau}\left\langle X_{\tau}^{\backslash}\right\rangle_{\lambda} \\
& =k^{-m \alpha / 2} \sum_{\tau} \sum_{n=0}^{\infty} \lambda^{n} \sum_{\Psi} \frac{1}{\Psi !}\left\langle X_{\tau}^{\rfloor}, \Phi_{\Psi}^{\backslash}\right\rangle_{0}
\end{aligned}
$$


by Lemma 5.4, where the first sum is taken over all $\tau=$ $\left(t_{1}, \ldots, t_{m}\right)$ with each $t_{i} \in C_{r_{i}}^{k}$ and the third sum is over all families $\Psi$ with $|\Psi|=n$ that connect $\tau$. By Lemma 5.4 for each $\tau$ the series $\sum_{n=0}^{\infty} \lambda^{n} \sum_{\Psi} \frac{1}{\Psi !}\left\langle X_{\tau}^{\backslash}, \Phi_{\Psi}^{\backslash}\right\rangle_{0}$ converges absolutely and uniformly on $\left[-C_{3}, C_{3}\right]$ and there are a finite number of $\tau$ in the sum, hence:

$$
\left\langle Y_{r_{1}}^{(k)}, \ldots, Y_{r_{m}}^{(k)}\right\rangle_{\lambda}=k^{-m \alpha / 2} \sum_{n=0}^{\infty} \lambda^{n} \sum_{\tau} \sum_{\Psi} \frac{1}{\Psi !}\left\langle X_{\tau}^{\downarrow}, \Phi_{\Psi}^{\dagger}\right\rangle_{0}
$$

and this series also converges absolutely and uniformly on $\left[-C_{3}, C_{3}\right]$.

\section{Lemma 5.7}

If $|\lambda|<C_{3}$, then each semi-invariant $\left\langle Y_{r_{1}}^{(k)}, \ldots, Y_{r_{m}}^{(k)}\right\rangle_{\lambda}$ is translation invariant.

\section{Proof}

If we shift a sequence $\left(r_{1}, r_{2}, \ldots, r_{m}\right)$ by vector $r$, then we get a new sequence $\left(r_{1}+r, r_{2}+r, \ldots, r_{m}+r\right)$. This shifts all sequences $\tau=\left(t_{1}, t_{2}, \ldots, t_{m}\right)$ and families $\Psi$ by vector $k r$ in (21) in Lemma 5.6. But it does not change the values of its addends because the field $\left\{X_{t} \mid t \in \mathbb{Z}^{v}\right\} \cup\left\{\Phi_{B} \mid B \in R\right\}$ is translation invariant (see Note 2 after Definition 2.3).

\section{Lemma 5.8}

For a fixed $t_{1} \in \mathbb{Z}^{v}$ denote:

$$
W\left(n, t_{1}, m\right)=\sum_{t_{2}, \ldots, t_{m} \in Z^{\nu}} \sum_{\Psi} \frac{1}{\Psi !}\left|\left\langle X_{\tau}^{\downarrow}, \Phi_{\Psi}^{\lfloor}\right\rangle_{0}\right|,
$$

where $\tau=\left(t_{1}, t_{2}, \ldots, t_{m}\right)$ and the second sum is taken over all families $\Psi$ with $|\Psi|=n$ that connect $\tau$. Then:

$$
W\left(n, t_{1}, m\right) \leq K(m)(n+1)^{m-1}\left(C_{1} C_{2}\right)^{n},
$$

where $K(m)=C(m, X)\left(C_{1}\right)^{m} m$ ! and $C(m, X)$ is defined in Lemma 4.1 .

\section{Proof}

For a non-zero addend in the sum, $|\Psi|=n$ and $\Psi$ connects $\left(t_{1}, t_{2}, \ldots, t_{m}\right)$, hence each of $t_{2}, \ldots, t_{m}$ is a vertex in the associated graph $G(\Psi)$. So there are at most $n+1$ choices for each of them. By Lemma 5.2, there are at most $\left(C_{2}\right)^{n}$ families $\Psi$ with $|\Psi|=n$ that connect $\tau$. Using also Lemma 4.1, we get:

$$
W\left(n, t_{1}, m\right) \leq(n+1)^{m-1}\left(C_{2}\right)^{n} C(m, X)\left(C_{1}\right)^{m+n} m !
$$

and

$$
W\left(n, t_{1}, m\right) \leq K(m)(n+1)^{m-1}\left(C_{1} C_{2}\right)^{n}
$$

\section{Lemma 5.9}

Suppose $|\lambda|<C_{3}$ and $r_{1}, \ldots, r_{m} \in \mathbb{Z}^{v}$. If $\alpha>v$ or $(\alpha=v$ and $m>2$ ), then:

$$
\lim _{k \rightarrow \infty}\left\langle Y_{r_{1}}^{(k)}, \ldots, Y_{r_{m}}^{(k)}\right\rangle_{\lambda}=0
$$

\section{Proof}

Case $m=1$ has been considered in Lemma 5.6.1). So we assume $m>1$. To estimate the semi-invariant we use the series (21) from Lemma 5.6 and consider the corresponding series of absolute values:

$$
A_{k}=k^{-m \alpha / 2} \sum_{n=0}^{\infty} B_{n, k}\left|\lambda^{n}\right|
$$

where $B_{n, k}=\sum_{\tau, \Psi} \frac{1}{\Psi !}\left|\left\langle X_{\tau}^{\backslash}, \Phi_{\Psi}^{\backslash}\right\rangle_{0}\right|$. Here the sum is taken over all sequences $\tau$ and families $\Psi$ as in (21).

Then $\left|\left\langle Y_{r_{1}}^{(k)}, \ldots, Y_{r_{m}}^{(k)}\right\rangle_{\lambda}\right| \leq A_{k}$ and it is sufficient to show that $\lim _{k \rightarrow \infty} A_{k}=0$.

Using the notation from Lemma 5.8, we have:

$$
B_{n, k} \leq \sum_{t_{1} \in C_{r}^{k}} W\left(n, t_{1}, m\right) .
$$

Here we omit the restriction that $t_{i} \in C_{r}^{k}$ for $i=2, \ldots$, $m$. By Lemma 5.8:

$W\left(n, t_{1}, m\right) \leq K(m)(n+1)^{m-1}\left(C_{1} C_{2}\right)^{n}$.

Since $t_{1} \in C_{r}^{k}$ there are $k^{v}$ choices for $t_{1}$. Since $C_{3} C_{1} C_{2}=\frac{1}{2}$, we have:

$$
\begin{aligned}
& A_{k} \leq k^{-m^{\alpha} / 2} \sum_{n=0}^{\infty}|\lambda|^{n} \sum_{t_{1} \in C_{r}^{k}} W\left(n, t_{1}, m\right) \\
& \leq k^{-m \alpha / 2} \sum_{n=0}^{\infty}\left(C_{3}\right)^{n} k^{v} K(m)(n+1)^{m-1}\left(C_{1} C_{2}\right)^{n} \\
& \leq k^{-m \alpha / 2} k^{v} K(m) \sum_{n=0}^{\infty}(n+1)^{m-1} \frac{1}{2^{n}} \\
& \leq k^{v-m} \alpha / 2 K(m) \frac{(m-1) !}{\left(1-\frac{1}{2}\right)^{m}} \\
& \leq k^{v-m \alpha / 2} K(m) 2^{m}(m-1) !
\end{aligned}
$$


For the last inequality we used Lemma A.1.1) from Appendix.

If $\alpha>v$ or $(\alpha=v$ and $m>2)$, then $v-m \alpha / 2<0$. So

$0 \leq \lim _{k \rightarrow \infty} A_{k} \leq K(m) 2^{m}(m-1) ! \lim _{k \rightarrow \infty} k^{\nu-m \alpha / 2}=0$

and $\lim _{k \rightarrow \infty} A_{k}=0$

\section{Corollary 5.1}

If $\alpha>v$, then for any $r \in \mathbb{Z}^{v}$ :

$$
\lim _{k \rightarrow \infty} \operatorname{Var}\left(Y_{r}^{(k)}\right)=0
$$

\section{Proof}

It follows from Lemma 5.9 because $\operatorname{Var}\left(Y_{r}^{(k)}\right)=\left\langle Y_{r}^{(k)}, Y_{r}^{(k)}\right\rangle_{\lambda}$.

\subsection{Finding the limiting covariances}

From here till the end of this section we consider only the case when $\alpha=v$ and $m=2$. Other cases are investigated earlier.

\section{Lemma 5.10}

Suppose $|\lambda|<C_{3}$. If $r_{1}, r_{2} \in \mathbb{Z}^{v}$ and $r_{1} \neq r_{2}$, then

$$
\lim _{k \rightarrow \infty}\left\langle Y_{r_{1}}^{(k)}, Y_{r_{2}}^{(k)}\right\rangle_{\lambda}=0
$$

\section{Proof}

We consider four cases.

Case 1: $r_{1}=\overline{0}$ and the first coordinate of $r_{2}$ is negative.

Denote $r=r_{2}$. Clearly, for any $t=\left(t_{1}, \ldots, t_{v}\right) \in C_{\overline{0}}^{k}$, we have: $0 \leq t_{1} \leq k-1$. Similarly, for any $s \in C_{r}^{k}, s_{1} \leq-1$. We introduce cross-sections of the cube $C_{\overline{0}}^{k}$ :

$$
D_{l}=\left\{t=\left(t_{1}, \ldots, t_{v}\right) \in C_{\overline{0}}^{k} \mid t_{1}=l\right\}, l=0,1, \ldots, k-1 .
$$

Clearly, $C_{\overline{0}}^{k}=\bigcup_{l=0}^{k-1} D_{l}$. Next we show:

if $t \in D_{l}, s \in C_{r}^{k}$ and $\Psi$ connects $(t, s)$, then $|\Psi| \geq l+1$. (25)

For $t \in D_{l}$ we have $t_{1}=1$. For $s \in C_{r}^{k}$ we have $s_{1} \leq-1$. So the distance between such $t$ and $\mathrm{s}$ is at least $l+1$.
If a family $\Psi$ connects $(t, s)$, then $|\Psi| \geq l+1$. This proves (25).

By Lemma 5.4:

$$
\begin{aligned}
& k^{v}\left\langle Y_{\overline{0}}^{(k)}, Y_{r}^{(k)}\right\rangle_{\lambda}=\sum_{t \in C_{\overline{0}}^{k}} \sum_{s \in C_{r}^{k}}\left\langle X_{t}, X_{s}\right\rangle_{\lambda} \\
& =\sum_{t \in C_{\overline{0}}^{k}} \sum_{s \in C_{r}^{k}} \sum_{n=0}^{\infty} \sum_{\Psi} \frac{\lambda^{n}}{\Psi !}\left\langle X_{t}, X_{s}, \Phi_{\Psi}^{\backslash}\right\rangle_{0},
\end{aligned}
$$

where the last sum is taken over all families $\Psi$ with $|\Psi|=n$ that connect $(t, s)$. have:

By (25) and since the series converges absolutely we

$k^{v}\left\langle Y_{\overline{0}}^{(k)}, Y_{r}^{(k)}\right\rangle_{\lambda}=\sum_{l=0}^{k-1} \sum_{t \in D_{l}} \sum_{s \in C_{r}^{k}} \sum_{n=0}^{\infty} \sum_{\Psi} \frac{\lambda^{n}}{\Psi !}\left\langle X_{t}, X_{s}, \Phi_{\Psi}^{\dagger}\right\rangle_{0}$

$=\sum_{l=0}^{k-1} \sum_{t \in D_{l}} \sum_{n=l+1}^{\infty} \sum_{\Psi} \sum_{s \in C_{r}^{k}} \frac{\lambda^{n}}{\Psi !}\left\langle X_{t}, X_{s}, \Phi_{\Psi}^{\backslash}\right\rangle_{0}$,

where $|\Psi|=n$ and $\Psi$ connects $(t, s)$.

For $\tau=(t, s)$ we have: $|\tau|=2, t \neq s$ and $\tau !=1$. Then by Lemma 4.1 for $m=2$ we get:

$\left|\left\langle X_{t}, X_{s}, \Phi_{\Psi}^{\backslash}\right\rangle_{0}\right| \leq C(2, X)\left(C_{1}\right)^{n+2} \Psi !$

Assume $\Psi$ and $t \in D_{l}$ are fixed, $|\Psi|=n$ and $\Psi$ connects $(t, s)$. Then the graph $G(\Psi)$ has at most $n+1$ vertices. At least $l+1$ of them have nonnegative first coordinates. In order for $\Psi$ to connect $(t, s)$, the point $s$ should be among the vertices of $G(\Psi)$ with negative first coordinates. Therefore there are at most $n+1-(l$ $+1)=n-l$ choices for $s$. So

$$
\begin{aligned}
& \sum_{\Psi} \sum_{s \in C_{r}^{k}}\left|\frac{\lambda^{n}}{\Psi !}\left\langle X_{t}, X_{s}, \Phi_{\Psi}^{\backslash}\right\rangle_{0}\right| \\
& \leq\left(C_{2}\right)^{n}(n-l) \frac{1}{\Psi !} C(2, X)\left(C_{1}\right)^{n+2} \Psi !|\lambda|^{n} \\
& \leq C(2, X)\left(C_{1}\right)^{2}(n-l)\left|\lambda C_{1} C_{2}\right|^{n} \leq K(2)(n-l) \frac{1}{2^{n}},
\end{aligned}
$$

where $K(2)=2 C(2, X)\left(C_{1}\right)^{2}$ as in Lemma 5.8.

$$
\begin{aligned}
& \sum_{n=l+1}^{\infty}(n-l) \frac{1}{2^{n}}=\frac{1}{2^{l+1}} \sum_{n=l+1}^{\infty}(n-l) \frac{1}{2^{n-l-1}} \\
& =\frac{1}{2^{l+1}} \sum_{k=1}^{\infty} k \frac{1}{2^{k-1}}=\frac{1}{2^{l+1}} \frac{1}{\left(1-\frac{1}{2}\right)^{2}}=\frac{1}{2^{l-1}}
\end{aligned}
$$

Since $D_{l}$ contains $k^{\nu-1}$ points, we have: 


$$
\begin{aligned}
& \left|\left\langle Y_{\overline{0}}^{(k)}, Y_{r}^{(k)}\right\rangle_{\lambda}\right| \leq k^{-v} K(2) \sum_{l=0}^{k-1} k^{v-1} \sum_{n=l+1}^{\infty}(n-l) \frac{1}{2^{n}} \\
& =\frac{K(2)}{k} \sum_{l=0}^{k-1} \frac{1}{2^{l-1}} \leq \frac{K(2)}{k} \frac{1}{2} \sum_{l=0}^{\infty} \frac{1}{2^{l}} \\
& =\frac{K(2)}{2 k} \cdot 2=\frac{K(2)}{k}
\end{aligned}
$$

Therefore

$$
\left|\left\langle Y_{\overline{0}}^{(k)}, Y_{r}^{(k)}\right\rangle_{\lambda}\right| \leq \frac{K(2)}{k}
$$

and

$\lim _{k \rightarrow \infty}\left\langle Y_{\overline{0}}^{(k)}, Y_{r}^{(k)}\right\rangle_{\lambda}=0$

Case 2: The first coordinate of $r_{1}$ is greater than the first coordinate of $r_{2}$. Then by Lemma 5.7, $\left\langle Y_{r_{1}}^{(k)}, Y_{r_{2}}^{(k)}\right\rangle_{\lambda}=\left\langle Y_{\overline{0}}^{(k)}, Y_{r_{2}-r_{1}}^{(k)}\right\rangle_{\lambda}$. Thus, Case 2 is reduced to Case 1 .

Case 3: The first coordinate of $r_{1}$ is less than the first coordinate of $r_{2}$. This is reduced to Case 2 by interchanging $r_{1}$ and $r_{2}$.

Case 4: The general case. Since $r_{1} \neq r_{2}$, they should differ in at least one coordinate, for example, in $j$-th coordinate. The proof is obtained by applying the proofs in Cases 1-3 to $j$-th coordinates instead of the first coordinates.

\subsection{Finding the Limiting Variances}

In the following theorem we derive explicit expressions for the limiting variances of $Y_{r}^{(k)}$. This theorem is interesting by itself and also becomes a part of the direct proof of the main theorem.

\section{Theorem 5.1}

Suppose $|\lambda|<C$, where $C=\min \left\{C_{3}, \frac{\sigma^{2}}{8 K(2) C_{1} C_{2}}\right\}$ and $\sigma^{2}$ is the variance of $X_{\overline{0}}$ with respect to $P_{0}$. Then for any $r \in \mathbb{Z}^{v}$ :

$$
\lim _{y \rightarrow \infty}\left\langle Y_{r}^{(k)}, Y_{r}^{(k)}\right\rangle_{\lambda}=\sum_{n=0}^{\infty} \lambda^{n} V_{n}=\sigma^{2}+\sum_{n=1}^{\infty} \lambda^{n} V_{n}>0 .
$$

Here the series converges absolutely and uniformly for $\lambda \in[-C, C]$ and:

$$
V_{n}=\sum_{t \in Z^{\nu}} \sum_{\Psi} \frac{1}{\Psi !}\left\langle X_{\overline{0}}, X_{t}, \Phi_{\Psi}^{\perp}\right\rangle_{0}
$$

where the second sum is taken over all families $\Psi$ with $|\Psi|=n$ that connect $(\overline{0}, t)$. For $n=0$, $V_{0}=\left\langle X_{\overline{0}}, X_{\overline{0}}\right\rangle_{0}=\sigma^{2}$.
Proof

Suppose $|\lambda|<C$. For any $s \in C_{\overline{0}}^{k}$ denote:

$\widetilde{W}(n, s, k)=\sum_{t \in C_{\overline{0}}^{k}} \sum_{\Psi} \frac{1}{\Psi !}\left\langle X_{s}, X_{t}, \Phi_{\Psi}^{\lfloor}\right\rangle_{0}$.

Here the second sum is taken over all families $\Psi$ with $|\Psi|=n$ that connect $(s, t)$.

If $n=0$, then in (28) we have $\Psi=\varnothing$, $\left\langle X_{s}, X_{t}, \Phi_{\Psi}^{\dagger}\right\rangle_{0}=\left\langle X_{s}, X_{t}\right\rangle_{0}$. For $t \neq s,\left\langle X_{s}, X_{t}\right\rangle_{0}=0$. So $\tilde{W}(0, s, k)=\left\langle X_{s}, X_{s}\right\rangle_{0}=\sigma^{2}$. Thus,

$\tilde{W}(0, s, k)=\sigma^{2}$.

Using Lemma 5.8 for $m=2$, we get:

$\left|V_{n}\right| \leq K(2)(n+1)\left(C_{1} C_{2}\right)^{n}$

$|\tilde{W}(n, s, k)| \leq K(2)(n+1)\left(C_{1} C_{2}\right)^{n}$.

For $n \geq 0$ and $k>2 n$ consider a cube $K_{n}^{k} \subset C_{\overline{0}}^{k}$ :

$K_{n}^{k}=\left\{s=\left(s_{1}, \ldots, s_{v}\right) \in \mathbb{Z}^{v} \mid n \leq s_{\mathrm{i}}<k-n\right.$ for each $\left.i=1, \ldots, v\right\}$.

Next we prove:

If $s \in K_{n}^{k}$, then $\tilde{W}(n, s, k)=V_{n}$.

\section{Proof of (32)}

Suppose $s \in K_{n}^{k}$. We will show that the sums (27) and (28) for $V_{n}$ and $\tilde{W}(n, s, k)$, respectively, contain equal addends.

Consider an addend $\left\langle X_{\overline{0}}, X_{t}, \Phi_{\Psi}^{\backslash}\right\rangle_{0}$ in (27); here $|\Psi|$ $=n$ and $\Psi$ connects $(\overline{0}, t)$. Translating all points by $s$, we get $\left\langle X_{s}, X_{t+s}, \Phi_{\Psi+\bar{s}}^{\perp}\right\rangle_{0}=\left\langle X_{\overline{0}}, X_{t}, \Phi_{\Psi}^{\backslash}\right\rangle_{0}$ and $B+\bar{s}$ connects $(s, t+s)$. Since $\|t-\overline{0}\| \leq|\Psi|=n$ and $s \in K_{n}^{k}$, we get $t+s \in C_{\overline{0}}^{k}$. Thus, for every addend in (27) there is an equal addend in (28). Similarly we can show that for every addend in (28) there is an equal addend in (27). The proof of (32) is completed.

Next we estimate the variance of $Y_{r}^{(k)}$, which equals $\left\langle Y_{r}^{(k)}, Y_{r}^{(k)}\right\rangle_{\lambda}$. By Lemma 5.7 and Lemma 5.6 we have: 


$$
\begin{aligned}
& \left\langle Y_{r}^{(k)}, Y_{r}^{(k)}\right\rangle_{\lambda}=\left\langle Y_{\overline{0}}^{(k)}, Y_{\overline{0}}^{(k)}\right\rangle_{\lambda} \\
& =k^{-v} \sum_{n=0}^{\infty} \lambda^{n} \sum_{s, t \in C_{\overline{0}}^{k}} \sum_{\Psi} \frac{1}{\Psi !}\left\langle X_{t}, X_{s}, \Phi_{\Psi}^{\dagger}\right\rangle_{0} \\
& =\sum_{n=0}^{\infty} \lambda^{n} k^{-v} \sum_{s \in C \overline{0}} \tilde{W}(n, s, k) .
\end{aligned}
$$

By (31) and because $C_{\overline{0}}^{k}$ contains $k^{v}$ points, we have:

$$
\begin{aligned}
& \left|\lambda^{n} k^{-v} \sum_{s \in C_{\frac{k}{0}}^{k}} \tilde{W}(n, s, k)\right| \leq|\lambda|^{n} k^{-v} \sum_{s \in C_{\frac{k}{0}}^{k}}|\tilde{W}(n, s, k)| \\
& \leq|\lambda|^{n} k^{-v} k^{v} K(2)(n+1)\left(C_{1} C_{2}\right)^{n} \leq K(2)(n+1) \frac{1}{2^{n}}
\end{aligned}
$$

Therefore the series (33) converges absolutely and uniformly for any $k$. We split the inner sum $\sum_{s \in C_{\overline{0}}^{k}} \tilde{W}(n, s, k)$ in two sums:

$$
U_{1}(n, k)=\sum_{s \in K_{n}^{k}} \tilde{W}(n, s, k)
$$

and

$$
U_{2}(n, k)=\sum_{s \in C_{\overline{0}}^{k} \backslash K_{n}^{k}} \tilde{W}(n, s, k) .
$$

So

$$
\begin{aligned}
& \left\langle Y_{r}^{(k)}, Y_{r}^{(k)}\right\rangle_{\lambda} \\
& =\sum_{n=0}^{\infty} \lambda^{n}\left[k^{-v} U_{1}(n, k)+k^{-v} U_{2}(n, k)\right]
\end{aligned}
$$

Since $K_{n}^{k}$ contains $(k-2 n)^{v}$ points, then by (32):

$$
U_{1}(n, k)=\sum_{s \in K_{n}^{k}} \tilde{W}(n, s, k)=(k-2 n)^{v} V_{n}
$$

and

$$
\lim _{k \rightarrow \infty} k^{-v} U_{1}(n, k)=\lim _{k \rightarrow \infty} \frac{(k-2 n)^{v}}{k^{v}} V_{n}=V_{n} .
$$

The difference of cubes $C_{\overline{0}}^{k} \backslash K_{n}^{k}$ contains $k^{v}-(k-2 n)^{v}$ points, so by (31) we have:

$$
\begin{aligned}
& \left|U_{2}(n, k)\right| \leq \sum_{s \in C_{\overline{0}}^{k} \backslash K_{n}^{k}}|\tilde{W}(n, s, k)| \\
& \leq K(2)(n+1)\left(C_{1} C_{2}\right)^{n}\left[k^{v}-(k-2 n)^{v}\right]
\end{aligned}
$$

and

$$
\begin{aligned}
& 0 \leq \lim _{k \rightarrow \infty}\left|k^{-v} U_{2}(n, k)\right| \\
& \leq K(2)(n+1)\left(C_{1} C_{2}\right)^{n} \lim _{k \rightarrow \infty} \frac{k^{v}-(k-2 n)^{v}}{k^{v}}=0
\end{aligned}
$$

By (34) and since the series converges uniformly for any $k$ :

$$
\begin{aligned}
& \lim _{k \rightarrow \infty}\left\langle Y_{r}^{(k)}, Y_{r}^{(k)}\right\rangle_{\lambda}=\sum_{n=0}^{\infty} \lambda^{n}\left[\lim _{k \rightarrow \infty} k^{-v} U_{1}(n, k)+\lim _{k \rightarrow \infty} k^{-v} U_{2}(n, k)\right] \\
& =\sum_{n=0}^{\infty} \lambda^{n} V_{n}=\sigma^{2}+\sum_{n=1}^{\infty} \lambda^{n} V_{n}
\end{aligned}
$$

because $V_{0}=\sigma^{2}$.

It remains to show that the limit is positive. By (30) and Lemma A.1.2) from Appendix:

$$
\begin{aligned}
& \sum_{n=1}^{\infty}\left|\lambda^{n} V_{n}\right| \leq \sum_{n=1}^{\infty}\left|\lambda^{n}\right| K(2)(n+1)\left(C_{1} C_{2}\right)^{n} \\
& =K(2) \sum_{n=1}^{\infty}(n+1)\left|\lambda C_{1} C_{2}\right|^{n} \\
& \leq K(2) \frac{2\left|\lambda C_{1} C_{2}\right|}{\left(1-\left|\lambda C_{1} C_{2}\right|\right)^{2}} \leq 8 K(2) C_{1} C_{2}|\lambda|<\sigma^{2} \\
& \text { because }\left|\lambda C_{1} C_{2}\right|<\frac{1}{2} \text { and }|\lambda|<\frac{\sigma^{2}}{8 K(2) C_{1} C_{2}}
\end{aligned}
$$

Therefore

$\sum_{n=1}^{\infty} \lambda^{n} V_{n}>-\sigma^{2}$ and $\sigma^{2}+\sum_{n=1}^{\infty} \lambda^{n} V_{n}>0$

\section{Proof of the Main Theorem (Theorem 3.1)}

Suppose $|\lambda|<C$, where $C$ is the constant from Theorem 5.1.

1. Case $\alpha>v$.

By Lemma 5.6.1) the limiting expectation of each $Y_{r}^{(k)}$ is 0 and by Corollary 5.1 the limiting variance is 0 . This proves part 1 of the theorem.

2. Case $\alpha=v$.

By Lemma 5.6.1) and Lemma 5.9 for $m \neq 2$ :

$$
\lim _{k \rightarrow \infty}\left\langle Y_{r_{1}}^{(k)}, \ldots, Y_{r_{m}}^{(k)}\right\rangle_{\lambda}=0
$$

By Lemma 5.10 the limiting covariances equal 0 and by Theorem 5.1 the limiting variance is positive. 
Thus, all of the limiting semi-invariants equal 0 , except the variance. Therefore the random variables $Y_{r_{1}}^{(k)}, Y_{r_{2}}^{(k)}, \ldots, Y_{r_{m}}^{(k)}$ converge in distribution as $k \rightarrow \infty$ to an independent multivariate normal random vector, due to Lemma 2.4. The statement about the variance of the limiting distribution follows from Theorem 5.1.

\section{Conclusion}

In this paper we introduce a concept of interaction model and prove a generalization of the central limit theorem to a random field in this model transformed by renormalization group. We show that as $k \rightarrow \infty$ the resulting random fields $Y_{r}^{(k)}$ converge in distribution to an independent random field with Gaussian distribution. We find the limits of all semi-invariants of $Y_{r}^{(k)}$ as $k \rightarrow \infty$ and apply Carleman theorem. In particular, we show that all the semi-invariants, except the variances, tend to 0 .

In Theorem 5.1 we give an explicit expression for the limiting variance. In order to find the limiting semi-invariants, we derive estimations of the semiinvariants of the original random field with respect to Gibbs measure.

We provide a more transparent proof under more general conditions for the inequality about the number of links in a set with a symmetric binary relation (Theorem 4.1). In this theorem and the lemmas about estimations of semi-invariants, as well as in the main theorem, we derive explicit expressions for the estimation constants.

A possible direction for future research is generalization of our theorem to other models in statistical mechanics.

\section{Appendix A}

\section{Lemma A.1}

If $0 \leq x<0.5$, then the following series converges absolutely for any $l=1,2, \ldots$ and

1) $\sum_{n=0}^{\infty}(n+1)^{l} x^{n} \leq \frac{l !}{(1-x)^{l+1}}$;

2) $\sum_{n=0}^{\infty}(n+1) x^{n} \leq \frac{2 x}{(1-x)^{2}}$.

\section{Proof}

1) We have

$$
(n+1)^{l} x^{n} \leq(n+1)(n+2) \ldots(n+l) x^{n}=\left(x^{n+l}\right)^{(l)} .
$$

Next:

$$
\sum_{n=0}^{\infty}\left(x^{n+l}\right)^{(l)}=\left(\sum_{n=0}^{\infty} x^{n}\right)^{(l)}=\left(\frac{1}{1-x}\right)^{(l)}
$$

because for $n<l$ we have $\left(x^{n}\right)^{(l)}=0$. It is easily proven by induction on $l$ that:

$$
\left(\frac{1}{1-x}\right)^{(l)}=\frac{l !}{(1-x)^{l+1}} .
$$

So the series (35) converges and satisfies the inequality.

2) If $l=1$, then

$$
\sum_{n=0}^{\infty}(n+1) x^{n}=\frac{1}{(1-x)^{2}}
$$

and

$$
\sum_{n=1}^{\infty}(n+1) x^{n}=\frac{1}{(1-x)^{2}}-1 \leq \frac{2 x}{(1-x)^{2}} .
$$

\section{Acknowledgment}

The authors are grateful to the Editor-in-Chief Carlo Bianca for very valuable help in producing this paper.

\section{Author's Contributions}

Each author contributed to the design of the research, the main results, proofs, and writing of the manuscript.

\section{Ethics}

This article is original and contains unpublished material. The corresponding author confirms that all of the other authors have read and approved the manuscript and no ethical issues are involved.

\section{References}

Bertini, L., E. Cirillo and E. Olivieri, 1999. Renormalization-group transformations under strong mixing conditions: Gibbsianness and convergence of renormalized interactions. J. Stat. Phys., 97: 831-915.

Bolthausen, E., 1982. On the central limit theorem for stationary mixing random fields. Ann. Probab., 10: 1047-1050.

Dobrushin, R.L., 1968. Gibbsian random fields for lattice systems with pairwise interactions. Funct. Anal. Applic., 77: 292-301. DOI: 10.1007/BF01075681 
Kachapova, F. and I. Kachapov, 2015. Application of semi-invariants to proof of the central limit theorem on a lattice. http://arxiv.org/pdf/1504.06935v2.pdf

Kadanoff, L.P., 1966. Scaling laws for Ising models near Tc. Physics, 2: 263-272.

Kadanoff, L.P., 2013. Relating theories via renormalization. Stud. Hist. Phil. Mod. Phys., 44: 22-39.

Kashapov, I.A., 1980. Justification of the renormalization-group method. Theory. Math. Phys., 42: 184-186. DOI: 10.1007/BF01032123

Lorinczi, J., Ch. Maes and K. Vande Velde, 1998. Transformations of Gibbs measures. Probab. Theory Related Fields, 112: 121-147.

Malyshev, V.A. and R.A. Minlos, 1991. Gibbs Random Fields: Cluster Expansions. 1st Edn., Kluwer, Dordrecht, ISBN-10: 079230232X, pp: 248.
Malyshev, V.A., 1980. Cluster expansions in lattice models of statistical physics and the quantum theory of fields. Russian Math. Surveys, 35: 1-62.

Newman, C.M., 1980. Normal fluctuations and FKG inequalities. Commun. Math. Phys., 74: 119-128.

Sinai, Y.G., 1976. Self-similar probability distributions. Theory Probab. Applic., 21: 64-80. DOI: $10.1137 / 1121005$

Wilson, K.G. and J. Kogut, 1974. The renormalization group and the epsilon expansion. Phys. Reports, 12: 75-199. DOI: 10.1016/0370-1573(74)90023-4 